Research Article

\title{
Investigation into Polyurethane at Varying Dose Rates of Ionizing Radiation for Clinical Application
}

\author{
Shelley L. Cooke $\mathbb{D}^{1}$ and Abby R. Whittington $\mathbb{D}^{1,2}$ \\ ${ }^{1}$ Department of Materials Science and Engineering, Virginia Polytechnic Institute and State University, Blacksburg, \\ VA 24061, USA \\ ${ }^{2}$ Department of Chemical Engineering, Virginia Polytechnic Institute and State University, Blacksburg, VA 24061, USA \\ Correspondence should be addressed to Abby R. Whittington; awhit@vt.edu
}

Received 25 April 2018; Accepted 16 July 2018; Published 1 October 2018

Academic Editor: Yves Grohens

Copyright (c) 2018 Shelley L. Cooke and Abby R. Whittington. This is an open access article distributed under the Creative Commons Attribution License, which permits unrestricted use, distribution, and reproduction in any medium, provided the original work is properly cited.

\begin{abstract}
Polyurethanes (PUs) are commonly used materials for medical devices. These devices are exposed repeatedly to radiation when patients undergo radiotherapy treatments. It has been found that peripherally inserted central catheters (PICCs) and central venous catheters (CVCs) fail at an increased rate $(14.7 \%$ and $8.8 \%$, respectively) when radiated. Currently, little research is available on increased failure seen in conjunction with radiation, but complex in vivo environments within a human patient make it difficult to isolate effects of individual variables. This research investigated effects of radiation in an aqueous environment to determine whether radiation combined with a mimicked in vivo environment is sufficient to change PU devices. The following dose rates were used in this study: $3.2 \mathrm{~Gy} \cdot \mathrm{min}^{-1}, 4.5 \mathrm{~Gy} \cdot \mathrm{min}^{-1}, 44 \mathrm{~Gy} \cdot \mathrm{min}^{-1}$, and $833 \mathrm{~Gy} \cdot \mathrm{min}^{-1}$. Samples were characterized in four main ways: cellular response, physical changes, chemical changes, and mechanical changes. Results reveal normal cellular response at all dose rates, indicating dose rate does not alter cellular adhesion or proliferation, and biocompatibility of the material is not being altered. Results from physical, chemical, and mechanical effects confirm that varying dose rates alone do not initiate material changes, which negates the hypothesis that varying dose rates of radiation contribute to the complications in PICC and CVCs.
\end{abstract}

\section{Introduction}

Polyurethane biomaterials are often studied for use in fabricating 3D tissue scaffolds, catheters, blood contact materials (heart valves and artificial veins), hospital bedding, injection molded implants, and other short-term implants [1-3]. Polyurethane catheters, specifically PICCs and CVCs, have been shown to fail at higher rates when exposed to radiotherapy in cancer patient $[4,5], 14.7 \%$ and $8.8 \%$, respectively [4]. Postinsertion complication rate of nonirradiated PICC lines is $30.4 \%$ [4-7]. Previous investigations in our lab (data not shown) have indicated that varying total therapeutic radiation doses at a constant dose rate does not alter PU enough to cause instability in an aqueous environment. However, altering radiotherapy dose rate is commonly used in treating different types of cancer or targeting specific tumor locations on the body [8] and may be a contributing factor to material stability in vivo [6]. Some researchers and clinicians have suggested that radiation should be applied prior to PICC line insertion to reduce complications believed to occur through radiation exposure (thrombosis, infection, mechanical occlusion/puncture, catheter movement, and extravasation), and this study aims to investigate whether radiation may be the cause of any complications [6,9-11].

The leading causes of PU catheter complications are infection and thrombosis (blood clotting). Radiation may play a role leading up to these biological responses and will be investigated using cell proliferation and adhesion studies on PU films [7]. Occlusion and puncture are mainly affected by the placement of the catheter in the body and may arise due to improper placement causing pinching of the catheter from arm movement or pinching between rib and clavicle, rupture due to pressure from liquid moving through 
catheter and the body's natural immune reaction to foreign materials causing catheter-associated thrombosis [9-12]. This study investigated these complications using biocompatibility tests combined with mechanical or chemical to provide indication of strength or chemical structure changes in the material which can aid in the movement of catheters contributing to complications [12]. Our current investigation does not focus on how material properties of catheters change only on whether or not they need to be removed.

The International Organization for Standardization (ISO 10555 and ISO 10993) and the United States Food and Drug Administration (FDA) regulations emphasize three major areas for catheter approval: toxicity/biocompatibility, mechanical properties, and thermal properties. All three were evaluated in this study in combination with chemical changes to determine if radiation causes significant changes leading to catheter failure $[13,14]$.

Currently, there is debate among clinicians on whether varying dose rate is actually effective in radiotherapy treatment. In order to clearly see changes between dose rates, a high sterilization dose was used to evaluate material changes as therapeutic radiation itself has been shown to not cause significant changes in PU materials under aqueous conditions chosen to mimic in vitro conditions [13, 15]. Polyurethane irradiated at sterilization doses or higher (World Health Organization standard for biomedical sterilization is $25 \mathrm{kGy}$ before material implantation) has shown significant alteration and should aid in determining whether varying dose rate also produces changes in polyurethane [16-20]. However, it is important to point out that this current study is focused on the changes occurring between dose rates.

The aqueous environment is a key factor in this research because it is an initial step in preclinical approval, mimicking an in vivo environment $[13,15]$. FDA and ISO guidelines for catheter preclinical studies require that catheter performance testing be simulated in in vivo conditions, such as at body temperature, and/or in water or saline environments $[15,21]$.

To apply different dose rates during radiotherapy, multiple LINAC instruments can be used [22]. Instrument energy level settings range between 6 and $22 \mathrm{MeV}$ (approximately $6-22 \mathrm{~Gy} \cdot \mathrm{min}^{-1}$ ), where low-range instruments use single 4 or $6 \mathrm{MeV}$ sources while high-range instruments may use multiple sources at once $[22,23]$. The low ranges are represented by both $3.2 \mathrm{~Gy} \cdot \mathrm{min}^{-1}$ and $4.5 \mathrm{~Gy} \cdot \mathrm{min}^{-1}$ dose rates that were evaluated in this study while the higher dose rates, $44 \mathrm{~Gy} \cdot \mathrm{min}^{-1}$ and $833 \mathrm{~Gy} \cdot \mathrm{min}^{-1}$, are the representative of multiple sources combined and high-dose rates [24]. The literature states that polyurethanes show alteration at high total doses; however, changes between varying dose rates have not been evaluated and may have implications in varying dose rates at low therapeutic doses as well $[16,19,25]$.

In this study, investigation was based on ISO standards to determine whether radiation changes in polyurethane samples were significant enough to cause samples to fall outside regulations set for device approval. This study addresses a concern voiced by clinicians that radiation may cause increased complications [4-7], and the principal goal was to isolate effects of radiation dose rates on polyurethane in a controlled aqueous environment.

\section{Materials and Methods}

2.1. Polyurethane Sample Preparation. Polyurethane pellets (polyether urethane; Texin 950) were gifted from Covestro ${ }^{\mathrm{TM}}$ AG (further information on this material is proprietary). Pellets were cryoground into powder using a three-step coolgrind-cool method repeated three times. Once sufficient polymer was ground, $2 \mathrm{~g}$ powder was pressed into films using a hot press at $365^{\circ} \mathrm{F}$ with $4,500 \mathrm{lbft}$ in -2 pressure for $2 \mathrm{~min}$. After $2 \mathrm{~min}$, a slight increase in pressure was used to remove any air bubbles, and samples were removed. Samples were then cut into $6.35 \mathrm{~mm}$ diameter circles with $0.03 \mathrm{~mm}$ thickness for testing (Figure 1). All samples were fabricated from the same batch of polyurethane pellets and pressed in the same day to eliminate batch-to-batch differences.

2.2. Material Radiation. Polyurethane films were irradiated in water at varying dose rates from different aged Cs-137 sources $(22,10,0.1$, and 1 years; Table 1$)$. Each dose rate was applied to a constant total dose of $25 \mathrm{kGy}$. Samples were irradiated at Oak Ridge National Laboratory using the High Flux Isotope Reactor, Gamma Irradiation Facility. Characterization methods were based on prior studies and ASTM procedure F561-13, which describes retrieval and recommended analysis of medical devices, associated tissues, and fluids. Once irradiated, films were cut into pieces for testing by various characterization methods described below. Sets of films stored in water at room temperature for the duration of the radiation experiment were compared to dry asfabricated. During control studies, it was found that the aqueous environment did not alter the polyurethane material resulting in all methods, specifically characterizing radiation effects. Films submersed in water showed no significant change from as-fabricated samples, and it can be concluded that any changes observed are the result of varying radiation dose. Preliminary results also confirm that overall sterilization dose significantly alters the polyurethane films (data not shown).

2.3. Cell Cytotoxicity and Proliferation. Polyurethane films were sterilized via $70 \%$ ethanol and then patted dry with Kimwipes $^{\mathrm{TM}}$. Samples were then immersed in alpha Minimum Essential Medium ( $\alpha$-MEM, Life Technologies) with $10 \%$ fetal bovine serum (FBS) and $1 \%$ penicillinstreptomycin for $12 \mathrm{hr}$. Soaked samples were placed in 48-well plates. Each sample was seeded with 5,000-mouse fibroblast cells (3T3, source ATCC) in a-MEM. This cell line was chosen because the fibroblast cells synthesize an extracellular matrix and collagen, common tissues found near polyurethane medical devices [26]. The plate was then incubated for $1 \mathrm{hr}$ at $37^{\circ} \mathrm{C}$ with $5 \% \mathrm{CO}_{2}$ to allow attachment. Once attached, cells were grown for 1,4 , and 7 days to determine fibroblast proliferation and cell cytotoxicity 


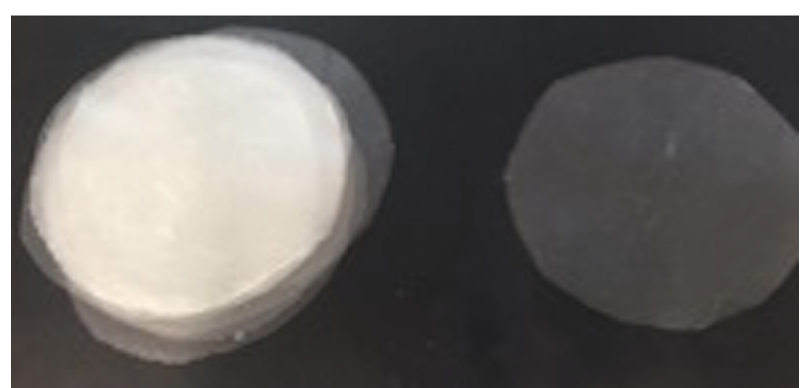

Figure 1: Fabricated polyurethane test films.

TABLE 1: Radiation sources and corresponding dose rates.

\begin{tabular}{lc}
\hline Age of source (\# of years) & Dose rate $\left(\mathrm{Gy} \cdot \mathrm{min}^{-1}\right)$ \\
\hline 22 & 3.2 \\
10 & 4.5 \\
1 & 44 \\
0.1 & 833 \\
\hline
\end{tabular}

within wells. A sample size of three was used for each time point and factor.

CellTiter $96^{\circledR}$ AQueous Non-Radioactive Cell Proliferation Assay (MTS, Promega, USA) was performed to determine cell proliferation, as previously described [27]. For adhesion studies, 1, 4, and 7 day polyurethane film samples were removed from wells and placed in new 48-well plates with $200 \mu \mathrm{L}$ of fresh media and $20 \mu \mathrm{L}$ MTS. All wells were incubated for $2 \mathrm{hr}$ at $37^{\circ} \mathrm{C}$ and $5 \% \mathrm{CO}_{2}$ to allow colorimetric reactions to occur with cells. All media were then transferred to a 96-well plate and read using a UV-VIS spectrophotometer at $490 \mathrm{~nm}$ (BioTek, Multi-Mode Plate Reader, Synergy Mx, USA). An empty polystyrene well was used as a positive control for all studies. A calibration curve was created using a standard cell count (102-106) and was used to determine the number of viable cells from each sample.

2.4. Size Exclusion Chromatography (SEC). Light scattering size exclusion chromatography (SEC; Agilent Technologies, USA) was used to determine polyurethane molecular weight. Samples $(n=3)$ were run in tetrahydrofuran (THF) at $1 \mathrm{~mL} \cdot \mathrm{min}^{-1}$ at $30^{\circ} \mathrm{C}$ on two Agilent PLgel $10 \mu \mathrm{m}$ MIXED-B columns connected in series with a Wyatt Dawn Helios 2 light scattering detector and a Wyatt Optilab Rex refractive index detector. An MW 21,000 polystyrene reference standard was used for calibration.

\subsection{Attenuated Total Reflectance Fourier Transform Infrared} Spectroscopy (ATR-FTIR). A Nicolet model 8700 Fouriertransform infrared (FTIR) instrument was employed to investigate polyurethane bond changes. FTIR spectra were recorded over a range of 500 to $4,500 \mathrm{~nm}$ with 126 scans per run and a resolution of $4 \mathrm{~cm}^{-1}$ sample size of 10 . A background scan was performed for each sample to remove baseline noise. Peak deconvolution was performed to quantify peak area under each curve. Percent change was calculated using Origin ${ }^{\circledR} 8.0$ graphing software. Seven major peaks were isolated for PU; all peak heights were measured and then averaged. A sample size of 10 films was used for each factor.

2.6. Dynamic Mechanical Analysis (DMA). Dynamic mechanical analysis (TA Q800) was used to investigate thermal degradation at different sample conditions. PU samples were heated from $-50^{\circ} \mathrm{C}$ to $140^{\circ} \mathrm{C}$ at a rate of $2^{\circ} \mathrm{C} \mathrm{min}{ }^{-1}$. Storage modulus, loss modulus, and Tan Delta were calculated with a sample size of three.

\subsection{Field-Emission Scanning Electron Microscopy (FESEM).} A LEO (Zeiss) 1550, field-emission environmental scanning electron microscope (FESEM), was used to determine microscopic surface differences between irradiated films. Films were sputter coated with gold using a high-vacuum Leica sputter coater. A sample size of two films was used, and representative images were taken at multiple locations on each film.

2.8. Statistical Analysis. Statistical analysis was performed for all tests, and corresponding graphs were created using Origin ${ }^{\circledR}$ 8.0. A $p$ value $<0.05$ was used in all $t$-tests, and ANOVA was computed to determine significant statistical differences.

\section{Results}

Polyurethane results are discussed in separate sections: cellular responses (adhesion and proliferation/migration), physical changes (FESEM and visual), chemical changes (FTIR-ATR and SEC), and thermal/mechanical responses (DMA). Results compare dose rates to determine if certain dose rates alter material properties more than others.

3.1. Cellular Responses. Mammalian cell response was investigated by looking at the cell number adhered on the films each day and then normalized to a tissue culture treated polystyrene control, and variability was calculated at 1,4 , and 7 days. Polyurethane samples displayed a decreased adherence after day 1 but had significantly lower growth than a polystyrene positive control (Figure 2). In day 1 (Figure 2), it was observed that polyurethane samples were $30 \%$ or less as adherent as polystyrene, while by day 7 , the polyurethane only had $\sim 10 \%$ adherence compared to the polystyrene-positive control. While both days 4 and 7 for the polyurethane had a significantly lower percentage $(18 \%$ and $17 \%$ less, respectively) of cells than day 1 , no statistical differences were seen between radiation doses within individual days, indicating that dose rate has no effect on cell adhesion but overall cells do not adhere to polyurethane films (Figure 2). This decrease in cellular attachment is not an indicator of cytotoxicity, as cells do proliferate in proximity to the films during this time. These results reveal a positive effect for this investigation since PICC and CVC lines are temporary implants, and adhesion is not desired. 


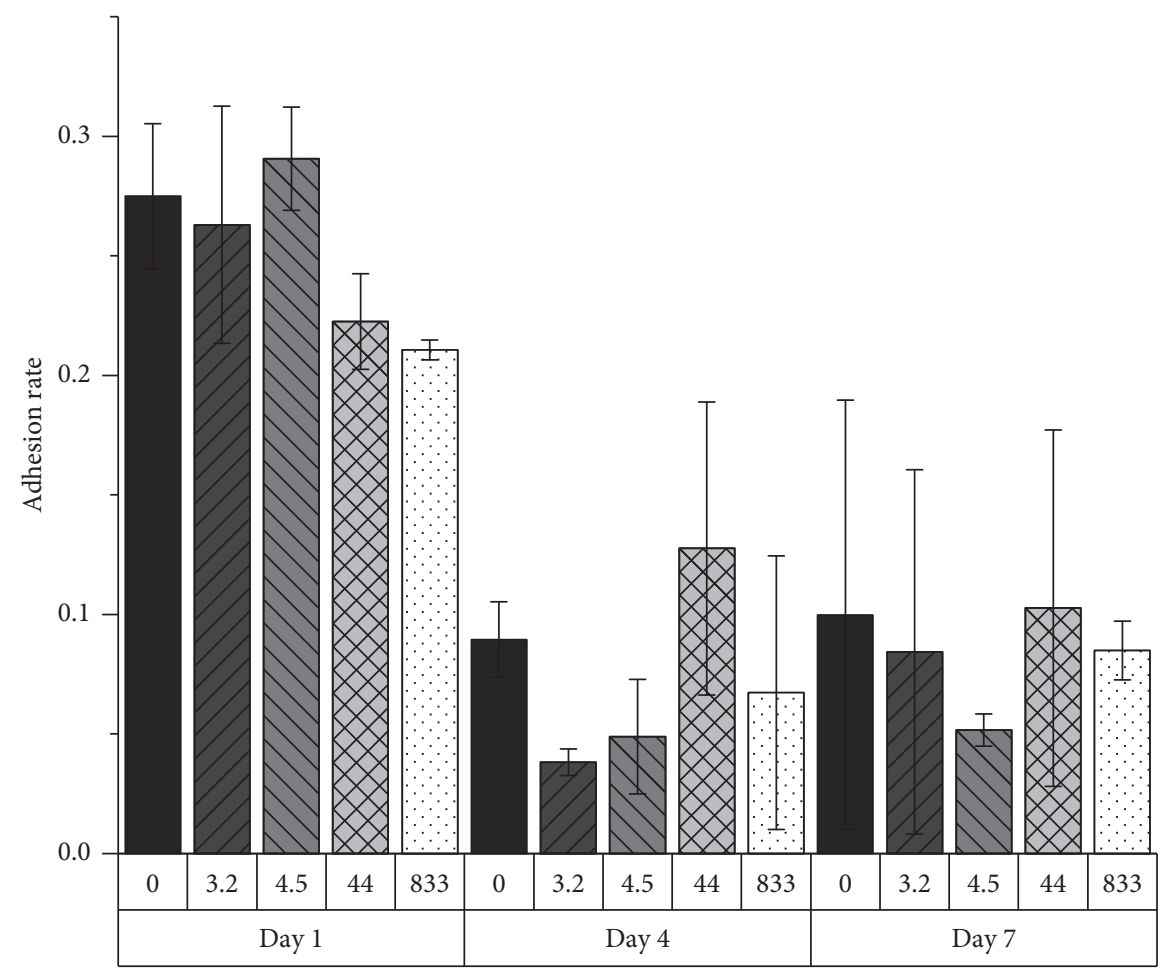

Figure 2: Polyurethane adhesion study of normalized cell attachment on samples $(n=3, p<0.05$, error bars depict standard deviations).

3.1.1. Physical Changes. Physical changes were detected using FESEM to determine if surface changes were occurring. Representative FESEM images (Figure 3) further elucidated microscopic differences. In all FESEM images, lines and minor surface particulates due to film pressing were seen, but little visual difference was found between dose rates.

ISO 10555 standards state that "with a minimum of $\times 2.5$ magnification the external surface of the effective length of the catheter shall appear free of extraneous matter" [13]. In this study, images showed that at $\times 2$ magnification, little to no differences were seen between dose rates. Surface changes indicate that dose rates do not affect polyurethane, and ISO guidelines for catheters were still met even with changes in dose rates.

\subsection{Chemical Changes from Irradiation}

3.2.1. Size Exclusion Chromatography (SEC). SEC was used to measure the number average molecular weight, polydispersity, and refractive index. SEC results revealed no significant difference in number average molecular weight or polydispersity (Figure 4; Table S1). However, significant changes were seen in refractive index (Figure 4). Refractive index results were significantly different between all dose rates except 44 and $833 \mathrm{~Gy} \cdot \mathrm{min}^{-1}$. Refractive index indicates a change in the speed in which light moved through the polyurethane and can indicate changes in composition (chemical structure/crystallinity) or purity (remnants of polymer, precipitates, modifiers, stabilizers, etc.) $[28,29]$. In the case of polyurethane samples for this study, the slight changes seen in refractive index are not due to molecular weight changes or crystallinity changes (see mechanical results) and are likely due to small impurities within the material. These impurities did not affect mechanical properties and are not likely to contribute to catheter movement or complication [10,12]. Methods used to view catheter movement (X-ray spectroscopy or electrocardiography) would also not be affected by changes in refractive index, and it can be concluded that although changes are seen, they would not cause failure in a clinical setting [10, 30, 31].

3.2.2. Fourier-Transform Infrared Spectroscopy. FTIR confirmed the results seen in SEC and indicated no major change in chemical structure. Seven peaks were isolated in the polyurethane spectra (data not shown). No new peaks were observed forming or disappearing, indicating that the polyurethane films do not undergo polymer backbone changes. The height of each peak can indicate a change in the number of bonds within the material and indicates that the material is remaining the same throughout all dose rate exposures (Table 2). Although slight variation is seen, FTIR results can be skewed due to the overlapping of peaks, and it is accepted that these slight changes are due to overlapping of peaks rather than significant changes in the material itself [32]. Control samples $(n=20)$ analyzed using FTIR were shown to have equal to or higher variations than each sample peak. This suggesting that variation within samples does not 

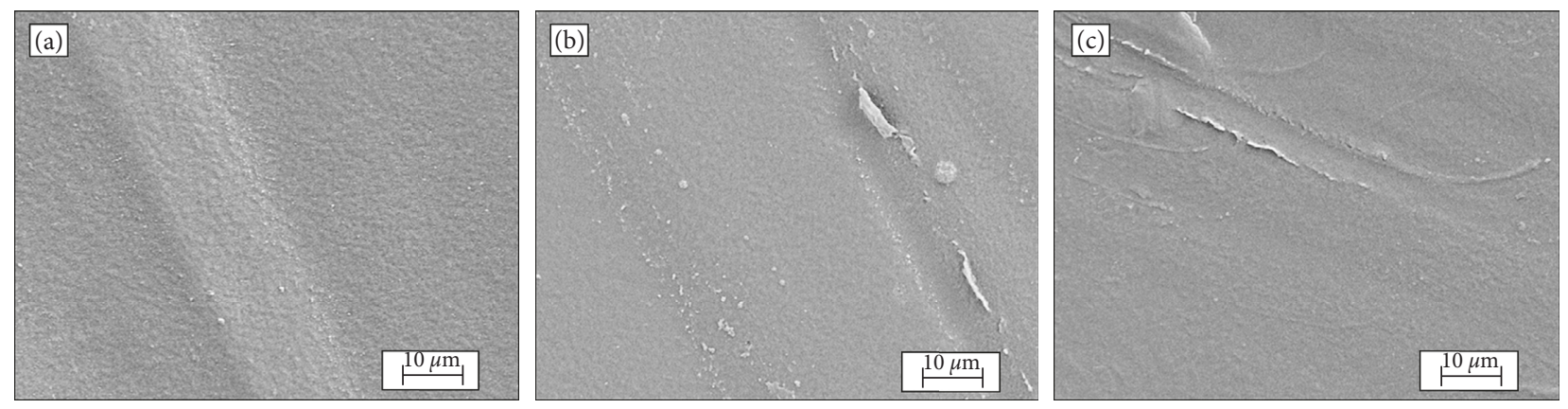

FIGURE 3: Field-emission scanning electron microscopy of polyurethane films sputter coated with gold at (a) $0 \mathrm{~Gy} \cdot \mathrm{min}^{-1}$, control (b) $3.2 \mathrm{~Gy} \cdot \mathrm{min}^{-1}$, and (c) $833 \mathrm{~Gy} \cdot \mathrm{min}^{-1}$ dose rates. Scale bars represent $10 \mu \mathrm{m}$.

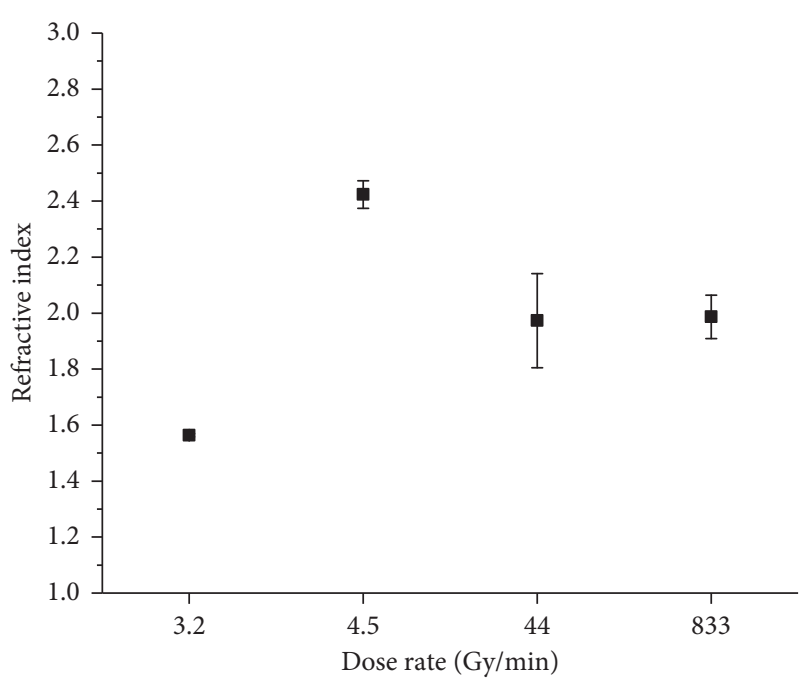

FIGURE 4: Refractive index changes show significant differences between all samples except between 44 and $833 \mathrm{~Gy} \cdot \mathrm{min}^{-1}(n=3$, $p<0.05$, error displayed in standard deviations).

indicate significant changes between varying dose rates but rather overlapping peaks and/or variation between sample films.

\subsection{Mechanical and Thermal Response}

3.3.1. Dynamic Mechanical Analysis (DMA). DMA was used to investigate mechanical and thermal changes in polyurethane samples. Two main types of information were collected from these tests: Tan Delta and storage modulus. Tan Delta is used to determine glass transition temperature and can indicate a shift in soft segments to hard segments [33]. Storage modulus is the elastic modulus indicating changes in energy storage and material elasticity [33]. Storage modulus was analyzed at room temperature $\left(25^{\circ} \mathrm{C}\right)$ and body temperature $\left(37^{\circ} \mathrm{C}\right)$ (Table 3$)$. No statistical differences were found between any of the dose rates evaluated. This was expected based on the chemical results previously discussed.

High variation was seen in the storage modulus data as well as within preliminary studies using nonirradiated, control samples. Power analysis was run using preliminary data to determine the number of samples for this study. This variation can be contributed largely to the variation in the material pellets themselves, combined with material fabrication (hand-pressed films), and would require further samples testing in the future. However, no trend was observed in the storage modulus data; it would have been expected for storage modulus to show an increase with increasing dose $[34,35]$. Due to the high variation within the groups, there was no true significance determined between the dose rates.

\section{Discussion}

This study found that varying dose rates alone in an aqueous environment does not alter cellular, physical, chemical, or mechanical properties. The belief that varying radiation dose rates causes changes in vivo is not supported by this study. These data are in agreement with Zhang et al., who investigated physical properties of catheters (fracture, leakage, and rupture) [6], as well as with previous research in our laboratory on therapeutic levels of radiation [36]. Radiation by itself or in an aqueous environment does not appear to cause the increased failure documented with use of PICC and CVC lines. It is important to note that polyether urethane used in this study is highly resistant to hydrolytic degradation [37]; control samples were not significantly different than the as-prepared polyurethane films (confirmed through FTIR, DMA, and SEC; data not shown), indicating that the aqueous environment employed in this study did not change properties of test films. The conditions in the study were selected to meet basic FDA regulations for testing in an in vitro environment.

Biocompatibility (ISO 10933) states that catheters shall be free of biological hazards. The first test listed is cytotoxicity, which states that cell culture techniques shall be used to determine cell death, inhibition of growth, colony formation, and other measured effects [14]. This study confirmed that cell death was not occurring using $24 \mathrm{hr}$ livedead testing (data not shown) and also confirmed that cell growth/proliferation was not inhibited. Cells grew as quickly as with the positive controls used. However, cellular adhesion shows a decrease over time, which is a positive outcome for catheter use since catheters during radiotherapy 
TABLE 2: FTIR Peak area data showing slight differences in bonds $(n=20)$.

\begin{tabular}{lccccccc}
\hline & $\mathrm{C}-\mathrm{O}-\mathrm{C}$, sym & $\mathrm{N}-\mathrm{H}, \mathrm{C}-\mathrm{N}$ & $\mathrm{N}-\mathrm{H}, \mathrm{C}-\mathrm{N}$ & $\mathrm{C}=\mathrm{O}$ stretch & ${\text { Sym. } \mathrm{CH}_{2}}$ & Asym. CH & $\mathrm{N}_{2} \mathrm{H}$ bond \\
\hline Gy·min & 1073 & 1216 & 1524 & 1700 & 2853 & 2933 & 3301 \\
3.2 & $0.44 \pm 0.01$ & $0.64 \pm 0.1$ & $0.72 \pm 0.01$ & $0.60 \pm 0.01$ & $0.49 \pm 0.01$ & $0.40 \pm 0.01$ & $0.25 \pm 0.0$ \\
4.5 & $0.54 \pm 0.04$ & $0.66 \pm 0.04$ & $0.72 \pm 0.03$ & $0.57 \pm 0.01$ & $0.38 \pm 0.01$ & $0.38 \pm 0.02$ & $0.23 \pm 0.01$ \\
44 & $0.51 \pm 0.04$ & $0.65 \pm 0.04$ & $0.70 \pm 0.02$ & $0.57 \pm 0.02$ & $0.46 \pm 0.04$ & $0.36 \pm 0.11$ & $0.24 \pm 0.01$ \\
833 & $0.51 \pm 0.01$ & $0.59 \pm 0.01$ & $0.71 \pm 0.01$ & $0.59 \pm 0.01$ & $0.45 \pm 0.02$ & $0.39 \pm 0.01$ & $0.25 \pm 0.0$ \\
\hline
\end{tabular}

TABLE 3: DMA results indicating significant changes between factors ( $n=3, p<0.05$, error displayed in standard deviations).

\begin{tabular}{lccc}
\hline $\mathrm{Gy} \cdot \mathrm{min}^{-1}$ & $\begin{array}{c}\text { Area }(\mathrm{Tan} \\
\text { Delta) }\end{array}$ & $\begin{array}{c}37^{\circ} \mathrm{C} \text { (storage, } \\
\mathrm{MPa})\end{array}$ & $\begin{array}{c}25^{\circ} \mathrm{C} \text { (storage, } \\
\mathrm{MPa})\end{array}$ \\
\hline 3.2 & $2.23 \pm 0.2$ & $51.15 \pm 17.8$ & $82.59 \pm 24.6$ \\
4.5 & $1.91 \pm 0.02$ & $70.20 \pm 33.9$ & $115.47 \pm 48.6$ \\
44 & $2.22 \pm 0.1$ & $56.22 \pm 29.2$ & $101.42 \pm 56.0$ \\
833 & $2.21 \pm 0.2$ & $44.05 \pm 19.5$ & $69.21 \pm 29.9$ \\
\hline
\end{tabular}

are temporary implants, and therefore, decreased cell adhesion is preferred.

Cellular response depends on a multitude of properties in an in vitro environment [38-40]. Some of these include biological factors (proteins/biological fluids), surface topography, surface chemistry, mechanical properties, and even absorption properties of the material itself $[37,39]$. Adhesion studies in this research found the majority of the cells only adhered for the first 24 hours. Polyurethane films were initially soaked in culture media before seeding the cells, so the initial contact between cell and material was between cells, and the media absorbed onto polyurethane implants. Cells do not begin to lay down a cellular matrix until several days later $[38,40]$. The culture media used in this study were used to promote cell growth. It was expected that cells would be initially interested in the material surface. Once the cells have thoroughly absorbed proteins on the surface of polyurethane samples, they came into contact with the polyurethane surface, and ultimately did not adhere to polyurethane films. Other research has discussed adhesion and suggested it is likely a combination of properties (chemistry, topography, and mechanics) that promote cell adhesion and makes it difficult to isolate effects [39].

Overall, cellular responses indicated positive results for temporary catheter implantation, and no changes were observed between any dose rates tested, signifying that varying dose rates did not alter polyurethane properties sufficiently to alter cellular response.

Microscopy results also confirmed the polymer stability seen in cellular, chemical, and mechanical tests. Visually, surfaces appeared similar, meeting the ISO standards of "free from extraneous matter". In relation to cellular response, surface topography plays a significant role in long-term cell adhesion while short-term cell adhesion $(24 \mathrm{hr})$ primarily responds to surface chemistry [38]. Surface topography did not change with varying dose rates, which supports the cellular adhesion results.

Along with surface topography, surface chemistry can affect both adhesion and proliferation. It was expected that chemical changes would reflect findings that dose rates do not cause significant alteration to polyurethane films. Sizeexclusion chromatography (SEC) was used to detect changes in molecular structure of the polyurethane polymer backbone. No changes in molecular weight or polydispersity indicate that dose rate is not causing backbone scission, and distribution of molecular mass remains the same. However, changes were seen in refractive index, which can be dependent on characteristics such as soft segments versus hard segments, chain extenders, and isocyanate used (methyl diphenyl diisocyanate, MDI or toluene diisocyanate, and TDI) [41-43]. Since the polyurethane used in this study is from the same batch of material, chain extenders and isocyanate should be constant, indicating that slight changes seen in refractive index are due to changes in soft versus hard segments. This indicates difference in amorphous and crystalline regions $[42,43]$. However, the Tan Delta shown in the DMA results indicated no significant shifts between soft and hard segments at any dose rate. It is possible that the small sample weight in SEC resulted in a poor representation of material composition due to increased variability. This is also seen in the $44 \mathrm{~Gy} \cdot \mathrm{min}^{-1}$ sample. However, refractive index results are within range of normal polyurethane materials, and it can be concluded that polyurethane films at all dose rates maintained chemical structure stability.

DMA results support clinical use of polyurethane films exposed at varying dose rates. Changes seen in DMA are regularly based on the ratio of hard segments to soft segments in the material [44]. Hard segments are isocyanate or diisocyanate linked to soft segments (ether-polyol) through a urethane linkage [45]. DMA shows no change in $\mathrm{Tg}$ or storage modulus at $25^{\circ} \mathrm{C}$ or $37^{\circ} \mathrm{C}$, indicating that ratios of hard segments to soft segments did not change supporting the absence of any chemical backbone changes seen in SEC data. Dose rates did not change material strength at room or body temperature, thus maintaining stability of catheters under clinical in vitro conditions.

\section{Conclusions}

Results from this study found that varying dose rates does not alter polyurethane material properties. Polyurethanes are common in clinical settings and have a high probability of being irradiated at various dose rates and different total doses in vivo. This study answered the question currently being posed by clinicians and researchers as to whether radiation is the main factor contributing to early failure in PICC and CVC lines; radiation alone does not cause material failure in polyurethane catheter applications. To the authors' 
knowledge, this research provides the first investigation into varying dose rates in an aqueous setting for clinical applications and has laid the groundwork to further investigate different medical devices and variables that may be exposed to radiation in vivo.

\section{Data Availability}

The data used to support the findings of this study are available from the corresponding author upon request.

\section{Conflicts of Interest}

The authors declare that there are no conflicts of interest regarding the publication of this article.

\section{Acknowledgments}

The authors would like to thank Geoffrey Deichert and Caleb Robison at Oak Ridge National Lab for conducting radiation exposures. The authors would also like to thank Jeffrey Weaver, Scott Radzinski, and Dr. John Matson for running size exclusion chromatography on polyurethane samples.

\section{Supplementary Materials}

Table S1: comparison of SEC results $(n=3$, error displayed in standard deviations). It includes numerical data collected from size exclusion chromatography. Results indicate no significant change in polydispersity or molecular weight $(p<0.05$, error in standard deviation). Figure S1: representative FTIR spectra of $0,3.2$, and $833 \mathrm{~Gy} / \mathrm{min}$ polyurethane samples. It compares resulting spectra for $0,3.2$, and $833 \mathrm{~Gy} / \mathrm{min}$ collected from Fourier-transform infrared spectroscopy. The spectra reveal little visual change between 3.2 and $833 \mathrm{~Gy} / \mathrm{min}$ but expected change from $0 \mathrm{~Gy} / \mathrm{min}$ due to overall sterilization dose. Major peak area and wavelength for all dose rates were further analyzed using peak deconvolution and discussed in Section 4.2 of the article. (Supplementary Materials)

\section{References}

[1] S. Lyu and D. Untereker, "Degradability of polymers for implantable biomedical devices," International Journal of Molecular Sciences, vol. 10, no. 9, pp. 4033-4065, 2009.

[2] R. J. Zdrahala and I. J. Zdrahala, "Biomedical applications of polyurethanes: a review of past promises, present realities, and a vibrant future," Journal of Biomaterials Applications, vol. 14, no. 1, pp. 67-90, 1999.

[3] A. Burke, Biomaterials: From Molecules to Engineered Tissues, Kluwer Academic/Plenum, New York, NY, USA, 2004.

[4] J. Bratton, P. Johnstone, and K. McMullen, "Outpatient management of vascular access devices in children receiving radiotherapy: complications and morbidity," Pediatric Blood and Cancer, vol. 61, no. 3, pp. 499-501, 2014.

[5] S. A. Jain, S. N. Shukla, S. S. Talati, S. K. Parikh, S. J. Bhatt, and V. Maka, "A retrospective study of central venous catheters GCRI experience," Indian Journal of Medical and Paediatric Oncology, vol. 34, no. 4, pp. 238-241, 2013.
[6] J. Zhang, S. Zhang, L. Li et al., "Effects of ionizing radiation on physical properties of peripherally inserted central catheter," PLoS One, vol. 11, no. 9, Article ID e0162837, 2016.

[7] T. Seckold, S. Walker, and T. Dwyer, "A comparison of silicone and polyurethane picc lines and postinsertion complication rates: a systematic review," Journal of Vascular Access, vol. 16, no. 3, pp. 167-177, 2015.

[8] H. B. Stone, C. N. Coleman, M. S. Anscher, and W. H. McBride, "Effects of radiation on normal tissue: consequences and mechanisms," The Lancet Oncology, vol. 4, no. 9, pp. 529-536, 2003.

[9] K. H. Polderman and A. R. Girbes, "Central venous catheter use," Intensive Care Medicine, vol. 28, no. 1, pp. 1-17, 2002.

[10] R. Gnannt, B. L. Connolly, D. A. Parra, J. Amaral, R. Moineddin, and A. S. Thakor, "Variables decreasing tip movement of peripherally inserted central catheters in pediatric patients," Pediatric Radiology, vol. 46, no. 11, pp. 1532-1538, 2016.

[11] V. Vidal, C. Muller, A. Jacquier et al., "Évaluation prospective des complications des PICCs," Journal de Radiologie, vol. 89, no. 4, pp. 495-498, 2008.

[12] A. Earhart, "Central lines: recognizing, preventing, and troubleshooting complications," Journal of the American Association, vol. 8, no. 11, 2013.

[13] International Organization for Standardization, 10555: Intravascular Catheters-Sterile and Single Use Catheters, International Organization for Standardization, Geneva, Switzerland, 2017.

[14] International Organization for Standardization, ISO 10993: Biological evaluation of Medial Devices, International Organization for Standardization, Geneva, Switzerland, 2010.

[15] Center for Devices and Radiological Health, Class II Special Controls Guidance Document for Certain Percutaneous Transluminal Coronary Angioplasty (PTCA) Catheters," in Medical Devices, United States Food and Drug Administration Silver Spring, MA, USA, 2010, https://www.fda.gov/ MedicalDevices/ucm225145.htm.

[16] N. Hirata, K. I. Matsumoto, T. Inishita et al., "Gamma-ray irradiation, autoclave and ethylene oxide sterilization to thermosetting polyurethane: sterilization to polyurethane," Radiation Physics and Chemistry, vol. 46, no. 3, pp. 377-381, 1995.

[17] H. Shintani and A. Nakamura, "Degradation and crosslinking of polyurethane irradiated by gamma-rays," Polymer Degradation and Stability, vol. 32, no. 2, pp. 191-208, 1991.

[18] H. Shintani and A. Nakamura, "Mechanism of degradation and crosslinking of polyurethane when irradiated by gammarays," Journal of Applied Polymer Science, vol. 42, no. 7, pp. 1979-1987, 1991.

[19] K. Gorna and S. Gogolewski, "The effect of gamma radiation on molecular stability and mechanical properties of biodegradable polyurethanes for medical applications," Polymer Degradation and Stability, vol. 79, no. 3, pp. 465-474, 2003.

[20] J. Haugen, M. Brunner, F. Pellkofer, J. Aigner, J. Will, and E. Wintermantel, "Effect of different $\gamma$-irradiation doses on cytotoxicity and material properties of porous polyetherurethane polymer," Journal of Biomedical Materials Research Part B: Applied Biomaterials, vol. 80B, no. 2, pp. 415-423, 2007.

[21] J. P. Santerre, K. Woodhouse, G. Laroche, and R. S. Labow, "Understanding the biodegradation of polyurethanes: from classical implants to tissue engineering materials," Biomaterials, vol. 26, no. 35, pp. 7457-7470, 2005. 
[22] E. B. Podgoršak, Radiation Oncology Physics, International Atomic Energy Agency, Vienna, Austria, 2005.

[23] M. J. Gazda and L. R. Coia, "Cancer management: a multidisciplinary approach: medical, surgical, and radiation oncology," in Principles of Radiation Therapy, Chapter 2, SCP Oncology Group, New York, NY, USA, 2005.

[24] E. J. Hall and D. J. Brenner, "The dose-rate effect revisited: radiobiological considerations of importance in radiotherapy," International Journal of Radiation Oncology Biology Physics, vol. 21, no. 6, pp. 1403-1414, 1991.

[25] S. Chandra, S. Sharma, H. Ali et al., "Effect of $\gamma$ irradiation on the impact response of rigid polyurethane foam," Journal of Polymer Engineering, vol. 36, no. 8, pp. 829-835, 2016.

[26] B. Alberts, A. Johnson, J. Lewi et al., "Molecular biology of the cell," in Fibroblasts and Their Transformations: The Connective-Tissue Cell Family, Garland Science, New York, NY, USA, 4th edition, 2002.

[27] Promega, 96 ${ }^{\circledR}$ AQueous One Solution Cell Proliferation Assay, Technical Bulletin, London, UK, 2005.

[28] H. Mark, "Purity and identity of polymers," Analytical Chemistry, vol. 20, no. 2, pp. 104-110, 1948.

[29] ASTM D542-14, Standard Test Method for Index of Refraction of Transparent Organic Plastics, ASTM International, West Conshohocken, PA, USA, 2014, http://www.astm.org.

[30] L. Kelly, "A practical guide to safe PICC placement," British Journal of Nursing, vol. 22, no. 5, pp. S13-S19, 2013.

[31] J. Jin, C. Chen, R. Zhao, A. Li, Y. Shentu, and N. Jiang, "Repositioning Techniques of malpositioned peripherally inserted central catheters," Journal of Clinical Nursing, vol. 22, no. 13-14, pp. 1791-1804, 2013.

[32] J. B. Huang and M. W. Urban, "Effect of overlapping bands on intensities in ATR FT-IR spectra," Applied Spectroscopy, vol. 46, no. 6, pp. 1014-1019, 1992.

[33] K. P. Menard and N. R. Menard, "Dynamic mechanical analysis in the analysis of polymers and rubbers," in Encyclopedia of Polymer Science and Technology, John Wiley \& Sons, Inc., Hoboken, NJ, USA, 2015.

[34] M. M. Ghobashy and Z. I. Abdeen, "Radiation crosslinking of polyurethanes: characterization by FTIR, TGA, SEM, XRD, and raman spectroscopy," Journal of Polymers, vol. 2016, Article ID 9802514, 9 pages, 2016.

[35] H. Shintani, H. Kikuchi, and A. Nakamura, "Effects of gamma-ray irradiation on the change of characteristics of polyurethane," Polymer Degradation and Stability, vol. 32, no. 1, pp. 17-30, 1991.

[36] S. L. Cooke and A. R. Whittington, "Influence of therapeutic radiation on polycaprolactone and polyurethane biomaterials," Materials Science and Engineering: C, vol. 60, pp. 78-83, 2016.

[37] E. M. Christenson, J. M. Anderson, and A. Hiltner, "Biodegradation mechanisms of polyurethane elastomers," Corrosion Engineering, Science and Technology, vol. 42, no. 4, pp. 312-323, 2007.

[38] K. Anselme, L. Ploux, and A. Ponche, "Cell/material interfaces: influence of surface chemistry and surface topography on cell adhesion," Journal of Adhesion Science and Technology, vol. 24, no. 5, pp. 831-852, 2010.

[39] X. Yao, R. Peng, and J. Ding, "Cell-material interactions revealed via material techniques of surface patterning," Advanced Materials, vol. 25, no. 37, pp. 5257-5286, 2013.

[40] C. J. Wilson, R. E. Clegg, D. I. Leavesley, and M. J. Pearcy, "Mediation of biomaterial-cell interactions by adsorbed proteins: a review," Tissue Engineering, vol. 11, no. 1-2, pp. 1-18, 2005.
[41] P. C. Painter and M. M. Coleman, Essentials of Polymer Science and Engineering, DEStech Publications, Inc., Lancaster, PA, USA, 2009.

[42] A. Puszka and A. Kultys, "The influence of soft segments on some properties of new transparent segmented polyurethanes," Polymers for Advanced Technologies, vol. 28, no. 12, pp. 1937-1944, 2017.

[43] S. Ioan, D. Macocinschi, D. Filip, and A. Taranu, "Specific refractive index increments of segmented poly(ester urethane) s," Polymer Testing, vol. 21, no. 7, pp. 757-762, 2002.

[44] C. Prisacariu, "Thermal behaviour of polyurethane elastomers," in Polyurethane Elastomers: From Morphology to Mechanical Aspects, pp. 61-101, Springer, Vienna, Austria, 2011.

[45] C. Prisacariu, "Chemistry of polyurethane elastomers," in Polyurethane Elastomers: From Morphology to Mechanical Aspects, pp. 1-22, Springer, Vienna, Austria, 2011. 

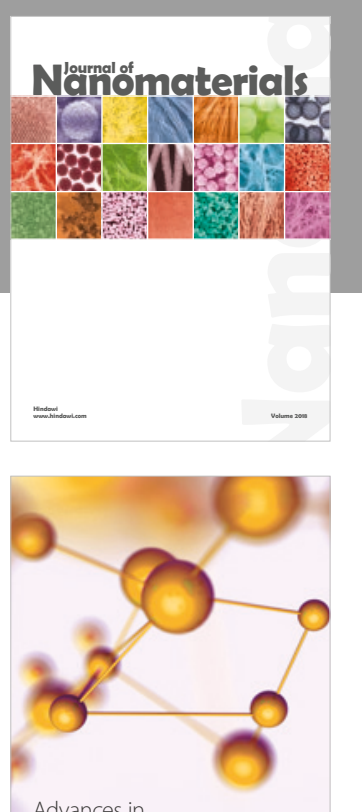

Physical Chemistry
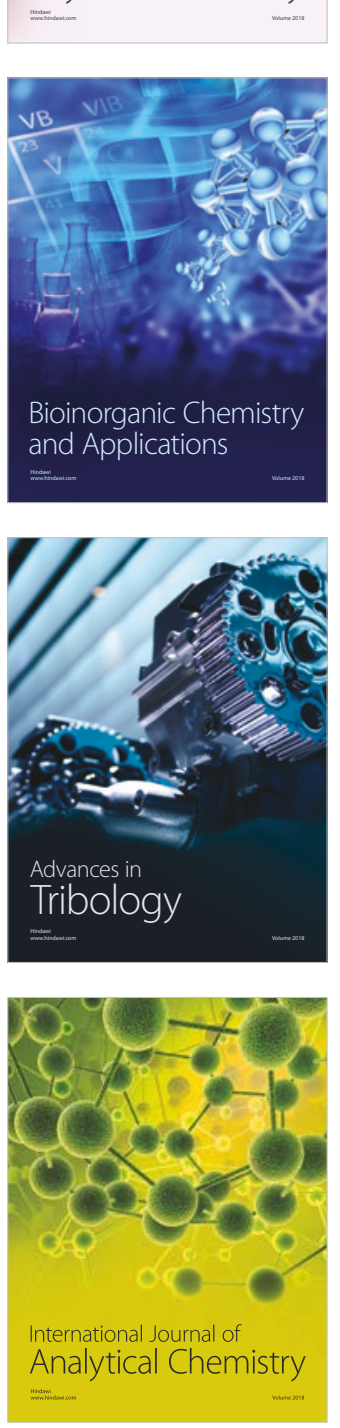

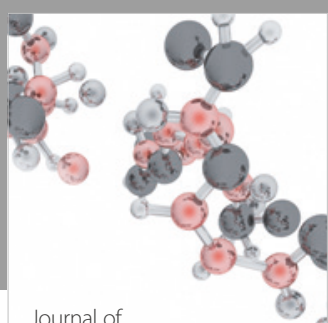

Analytical Methods

in Chemistry

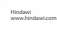

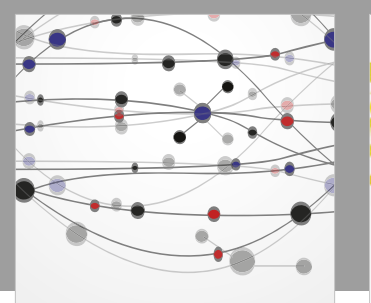

The Scientific World Journal

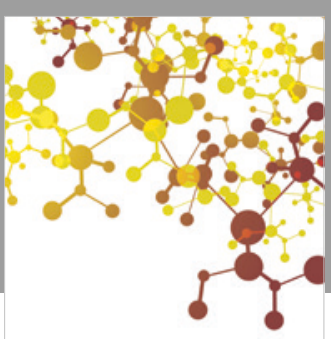

Journal of

Applied Chemistry
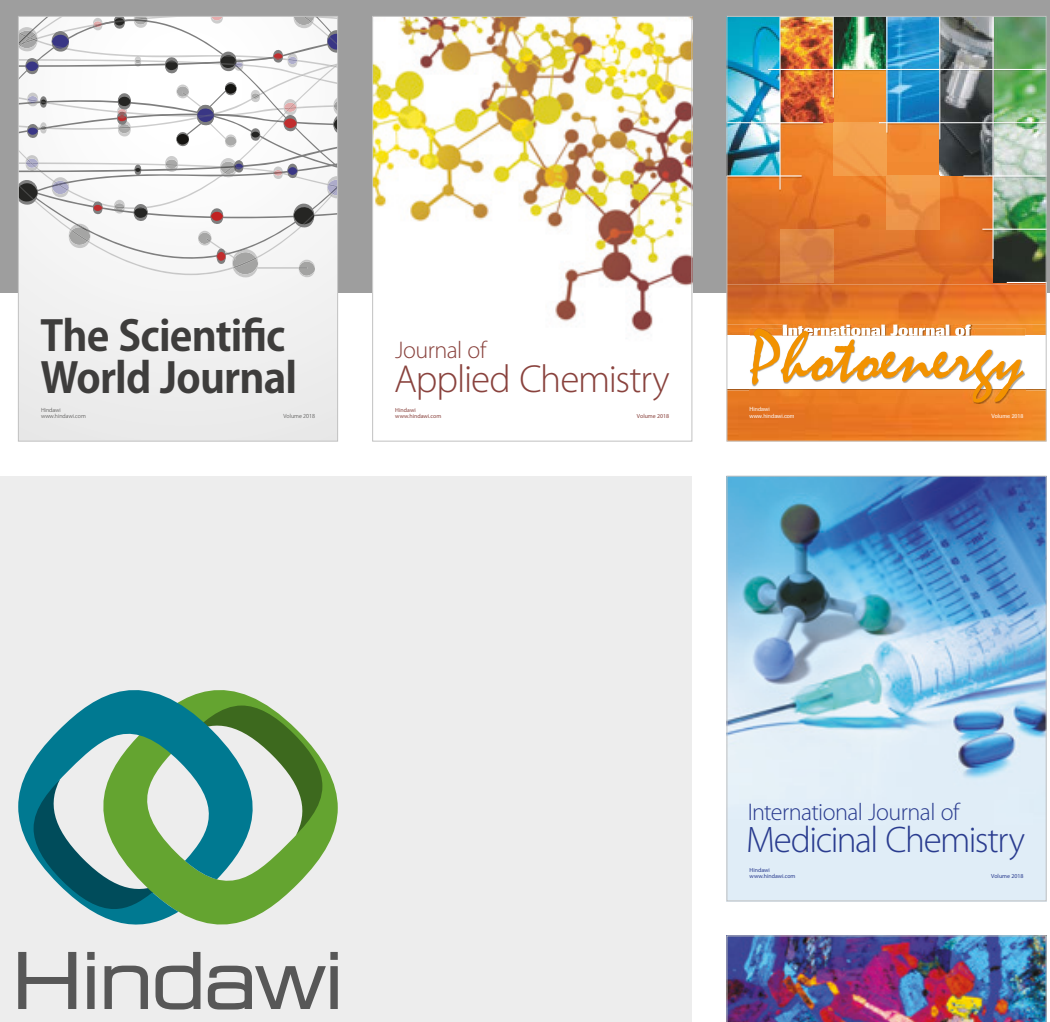

Submit your manuscripts at

www.hindawi.com
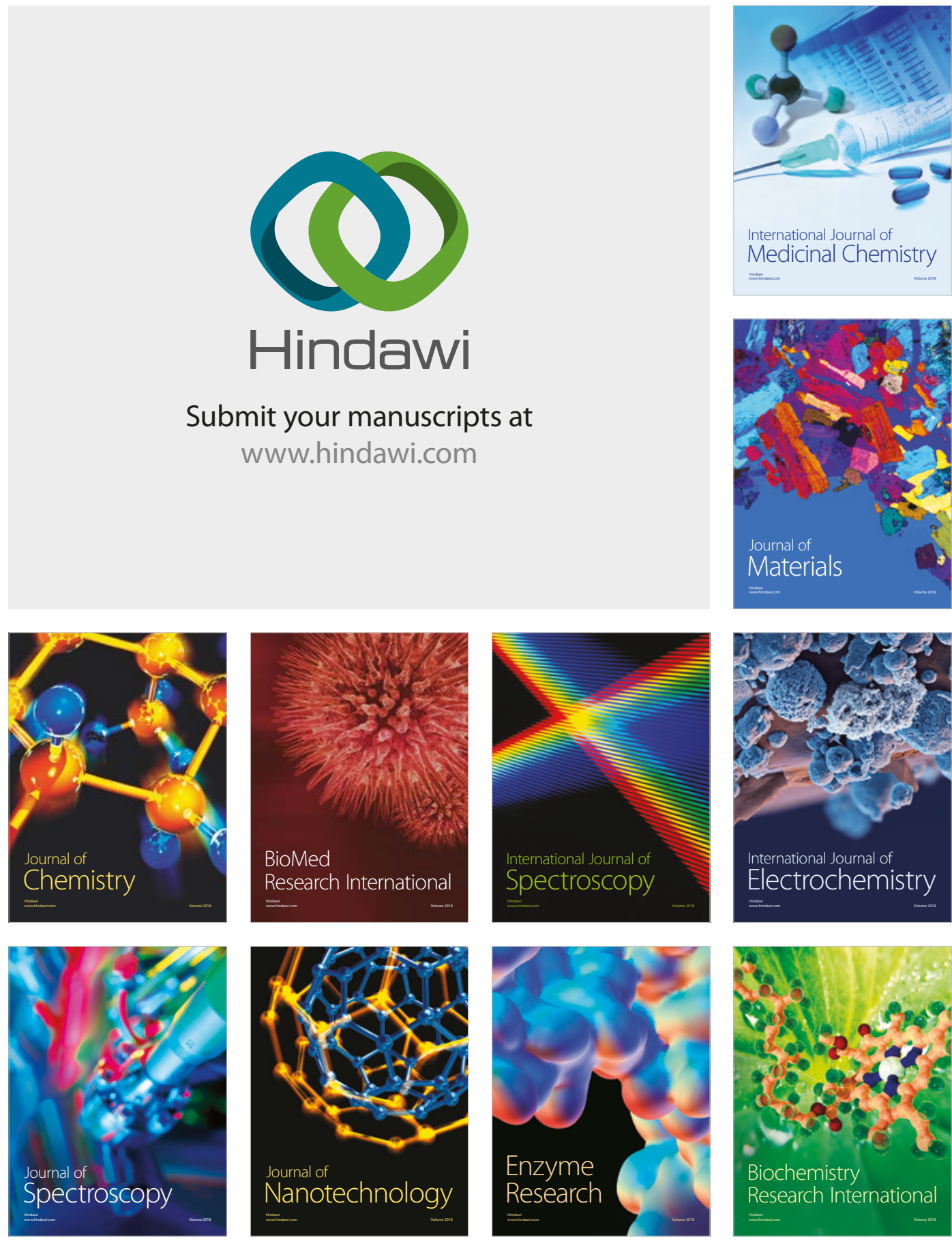
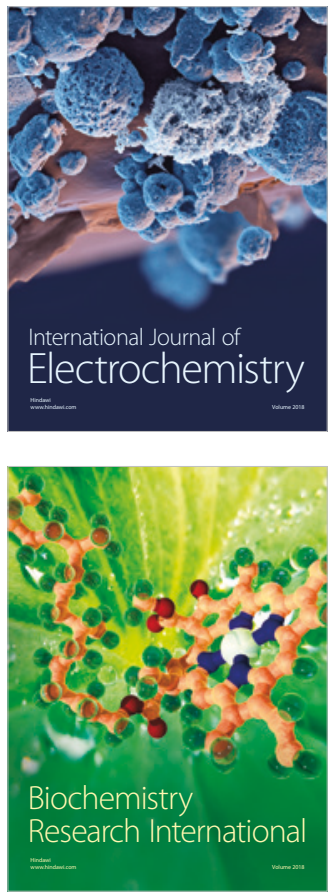